\title{
DPD status and fluoropyrimidines-based treatment: high activity matters too
}

Emmanuel Chamorey ${ }^{1 *}$ (D) Eric Francois ${ }^{2}$, Marie-Christine Etienne ${ }^{3}$, Jean-Marc Ferrero ${ }^{2}$, Frederic Peyrade ${ }^{2}$, Emmanuel Barranger ${ }^{4}$, Alexandre Bozec ${ }^{5}$, Rémy Largillier ${ }^{2}$, Ophelie Cassuto ${ }^{2}$, Julien Viotti ${ }^{1}$, Renaud Schiappa ${ }^{1}$ and Gérard Milano ${ }^{3}$

\begin{abstract}
Background: Dihydropyrimidine dehydrogenase (DPD) status is an indicator of a marked risk for toxicity following fluoropyrimidine (FP)-based chemotherapy. This notion is well-established for low DPD status but little is known about the clinical impact of high DPD activity. This study examined the possible link between high intrinsic lymphocytic DPD activity and overall survival, progression free survival and response to FP-based treatment in patients treated in our institution.

Methods: Lymphocytic DPD activity was assessed in a group of 136 patients receiving FP-based chemotherapy from 2004 to 2016. There were 105 digestive (77.2\%), 24 breast (17.6\%) and 7 head and neck cancers (5.2\%). Cox or logistic regression models were applied with adjustment on all confounding factors that could modify OS, PFS or response. All models were stratified on the three cancer locations. A cut-off for DPD activity was assessed graphically and analytically.

Results: An optimal cut-off for DPD activity at $0.30 \mathrm{nmol} / \mathrm{min} / \mathrm{mg}$ protein was identified as the best value for discriminating survivals and response. In multivariate analysis, individual lymphocytic DPD activity was significantly related to overall survival ( $p=0.013$; HR: 3.35 C195\%[1.27-8.86]), progression-free survival $(p<0.001$; HR: 3.15 C195\%[1.75-5.66]) and response rate ( $p=0.033 ;$ HR: 0.33 C195\%[0.12-0.92]) with a marked detrimental effect associated with high DPD activity.
\end{abstract}

Conclusions: DPD status screening should result in a two-pronged approach with FP dose reduction in case of low intrinsic DPD and, inversely, an increased FP dose for high intrinsic DPD. In a context of personalized FP-based treatment, this innovative strategy needs to be prospectively validated.

Keywords: Dihydropyrimidine dehydrogenase, Capecitabine, 5-fluorouracil, Digestive neoplasms, Breast neoplasms, Head and neck neoplasms

\footnotetext{
* Correspondence: emmanuel.chamorey@nice.unicancer.fr

${ }^{1}$ Centre Antoine-Lacassagne, Epidemiology and Biostatistics Unit, Université Côte d'Azur, 33 Avenue de Valombrose, 06189 Nice Cedex 2, Nice, France

Full list of author information is available at the end of the article
}

(c) The Author(s). 2020 Open Access This article is licensed under a Creative Commons Attribution 4.0 International License, which permits use, sharing, adaptation, distribution and reproduction in any medium or format, as long as you give appropriate credit to the original author(s) and the source, provide a link to the Creative Commons licence, and indicate if changes were made. The images or other third party material in this article are included in the article's Creative Commons licence, unless indicated otherwise in a credit line to the material. If material is not included in the article's Creative Commons licence and your intended use is not permitted by statutory regulation or exceeds the permitted use, you will need to obtain permission directly from the copyright holder. To view a copy of this licence, visit http://creativecommons.org/licenses/by/4.0/ The Creative Commons Public Domain Dedication waiver (http://creativecommons.org/publicdomain/zero/1.0/) applies to the data made available in this article, unless otherwise stated in a credit line to the data. 


\section{Background}

Fluoropyrimidines (FP) are antimetabolite drugs widely used for treatments of many cancers, including digestive, breast, and head and neck locations. FP are delivered as intravenous 5-FU or orally with capecitabine which is a prodrug converted to 5-FU inside target cells. The estimated frequency of significant FP-related toxicity is around $10 \%$ including 0.1 to $1.0 \%$ of lethal ones [1]. Dihydropyrimidine dehydrogenase (DPD) is the main enzyme responsible for FP catabolism. Population studies $[2,3]$ on lymphocyte DPD distribution indicate a Gaussian curve of DPD activity distribution including both low and high values. The elucidation of genetic mechanisms underlining DPD variability is increasingly being appreciated [4]. Loss of DPD activity evidenced by phenotypic and/or genotype screening has proven its predictive clinical value in identifying patients at risk for toxicity [1, 3, 5-9]. A recent multicenter prospective study has shown the feasibility of DPYD genotypeguided dose individualization before FP treatment to reduce toxicity [10].

High intrinsic DPD activity results in an accelerated FP degradation [11]. This may translate into a variable marked loss of antitumor efficacy due to the relative lack of FP available for the activation routes at target cell level, and also into a pharmacokinetic effect with a relative decrease in circulating FP [11-14]. The clinical impact of high intrinsic DPD activity on FP-based treatment outcome remained to be investigated. This retrospective monocentric study aimed to fill this gap and examined the link between lymphocytic DPD activity and overall survival, progression-free survival and response rate in a cohort of FP-treated representative patients tested for DPD activity and treated by FP-based chemotherapy in a single institution from 2004 to 2016. The covered cancer locations (digestive tract, breast, head and neck) correspond to the true life of FP-based treatment and were considered in the present study in order to be representative of the routine use of FP in cancer treatment.

\section{Methods}

\section{Patients}

One hundred and thirty-six patients treated by FP-based chemotherapy and assessed for intrinsic DPD activity were included in this retrospective monocentric cohort study covering a twelve-year period from 2004 to 2016. The study comprised of 78 females $(57.3 \%)$ and 58 males (42.6\%); 105 digestive cancers (77.2\%), 24 breast cancers (17.6\%) and 7 head and neck cancers (5.2\%). All patients were consecutively treated at the Centre Antoine Lacassagne (Comprehensive Cancer Center, Nice, France). Intrinsic DPD activity ( $\mathrm{nmol} / \mathrm{min} / \mathrm{mg}$ protein) in peripheral blood mononuclear cells was assessed as previously described by Harris and coll $[15,16] .$. In brief, DPD activity (blood sample taken between 9:00 and 11: $00 \mathrm{am}$ ) was measured in mononuclear cells using a radioenzymatic assay $(2.5 \mathrm{mM} \mathrm{MgCl} 2,250 \mu \mathrm{M} \mathrm{NADPH}$, $20 \mu \mathrm{M} 14 \mathrm{C} 5-\mathrm{FU})$ with separation of 14C5-FU from 14C5-FUH2 by High-Performance Liquid Chromatography (HPLC) coupled with a radiodetector. DPD testing was performed before start of FP-treatment or after initiation of FP-treatment. This study was declared to the Commission Nationale Informatique et Liberté (CNIL $\left.\mathrm{N}^{\circ} 17,002\right)$ and all patients agreed that their data could be used for retrospective biomedical study.

\section{Statistics}

OS was defined as the time from FP initiation to death. PFS was defined as the time from FP initiation to death or either distant, local or metastatic progression as defined by the RECIST version 1.1 criteria. Patients showing no event (death or progression) or lost to follow-up were censored at the date of their last contact. In our study, response to therapy was evaluated prospectively using RECIST version 1.1 criteria's when possible. Otherwise, observed complete response during FP treatment (CR) was defined as disappearance of all target lesions after chemotherapy alone or multimodal treatment including chemotherapy, radiotherapy and/or local ablative therapy. Statistical comparisons were performed using Cox regression model for survival data or logistic regression model for response analysis. Others quantitative data were compared by using Student T test or Wilcoxon test when appropriated and qualitative data were analyzed by using Fisher exact test. In order to avoid all confounding factors that could modify OS, PFS or Response, all multivariate models were stratified for cancer locations (digestive, breast and head and neck) and adjusted for all variables associated with $p<0.10$ on univariate analysis. Variables analyzed were defined as follow: DPD activity ( $\mathrm{nmol} / \mathrm{min} / \mathrm{mg}$ protein), sex (male/ female), disease severity (local/advanced), DPD prescription before FP start or during FP treatment, other chemotherapy treatment before FP (no/yes), type of FPbased treatment (Capecitabine, 5-FU, Both alternatively), observed FP-based toxicity $>$ grade 3 or 4 by using CTCAE V4.0 or toxicity inducing FP-based treatment stop (no/yes), any surgery associated to FP (no/yes), any radiotherapy associated to $\mathrm{FP}$ (no/yes), observed complete response during FP treatment (no/yes), any local, loco regional or metastatic recurrence observed (no/yes), age at FP-based treatment (years), number of chemotherapy lines including FP treatment $(\mathrm{N})$, number of FP cycles (N). Proportional hazards were tested for all variables entered in Cox models using graphical Schoenfeld residuals and statistical test. Sensitivity analyses for all tested models were performed firstly by removing 
outliers when indicated and secondly by testing the same multivariate models only for the 105 digestive cancer patients. Statistical analyses were two-sided and were performed using R-3.2.3 for Windows.

\section{DPD cut-off assessment}

The cut-off for DPD activity predicting OS, PFS and response was evaluated graphically by using inflection points of the smoothing spline curve fit to DPD activity (see Figs. S1, S2 and S3 in supplementary data showing smoothing spline fit to DPD activity versus OS, PFS and Response) and confirmed by using statistical rules assessed using $\mathrm{R}$ function "bestcut2" for survival data model as well as the $\mathrm{R}$ function "optimal.cutpoints" which established the optimal cut-point for a logistic regression model.

\section{Results}

DPD activity followed a Gaussian distribution with mean $(+/-\mathrm{SD})$ at $0.21 \mathrm{nmol} / \mathrm{min} / \mathrm{mg}(+/-0.10)$ and quartiles [0.002-0.15-0.20-0.28-0.48]. The best identified cut-off for DPD activity predicting OS, PFS and response was $0.30 \mathrm{nmol} / \mathrm{min} / \mathrm{mg}$ protein; this DPD value was included in all univariate and multivariate analysis. This cut-off is the most relevant point according to graphical and analytical assessment and is corresponding to almost the third quartile of the distribution of DPD activity (i.e.: $0.28 \mathrm{nmol} / \mathrm{min} / \mathrm{mg}$ protein). Twenty-five patients (18.4\%) presented DPD activity $\geq 0.30$ and $111(81.6 \%)$ presented DPD activity $<0.30 \mathrm{nmol} / \mathrm{min} / \mathrm{mg}$ protein. Most of the DPD activity assessments were performed before the beginning of FP-based treatment (108; 79.4\%). No difference was observed in DPD activity when measurement was performed before or during FP-based treatment $(p=0.59$, Table 1$)$. Ninety six patients $(70.5 \%)$ had advanced disease, including 66 with loco-regional and 30 with metastatic disease. Mean age was 64.2 years $(+/-$ 12.4). Thirty-eight patients (27.9\%) received capecitabine based treatment, 70 (51.5\%) received 5-Fluorouracil based treatment (5-FU) associated with folinic acid and $28(20.6 \%)$ received both treatments alternatively. Median number of total FP cycles was 8 (range: $1-48$ ); 33 patients $(24.3 \%)$ received other chemotherapy prior to FP-based treatment. Median number of chemotherapy lines (FP or not FP) was 1 (range 1-12). One hundred patients $(73.5 \%)$ had surgery associated with FP-based treatment and 77 (56.6\%) received radiotherapy. Seventy-six patients (55.9\%) showed complete response during FP-based treatment. Recurrence was observed in 77 (56.6\%) patients and 58 patients (42.6\%) presented FP-related toxicity. Table 1 brings more detailed information regarding patient and treatment characteristics versus DPD activity levels (low versus high, ie: $<0.30$ versus $\geq 0.30$ ) it shows that observed complete response during FP treatment ( $p=0.032$, Fisher exact test), was significantly linked to DPD activity.

Univariate analyses for overall survival, progressionfree survival and response rate are summarized in Table 2. OS, PFS and response rate were significantly related to DPD activity $(p=0.0018,0.0016$ and 0.022 , respectively) with, a marked detrimental effect linked to high DPD activity. Survival curves for OS and PFS are depicted in Fig. 1 a and b. Table 2 shows that disease severity $(p=0.046)$, surgery associated to $\mathrm{FP}(p=0.030)$, observed complete response during FP treatment $(p<$ $0.0001)$, any recurrence $(p=0.0026)$ and age $(p=0.0062)$ were significantly associated with OS. Disease severity at initial diagnostic $(p=0.0079)$, other chemotherapy before FP $(p=0.0022)$, age $(p=0.0069)$ and number of FP cycle $(p<0.001)$ were significantly related to PFS. Also illustrated from Table 2, disease severity at initial diagnostic $(p=0.008)$ and other chemotherapy before FP $(p=0.00069)$ were found significantly related to response.

In multivariate analysis (Table 3 ) including all variables with $p<0.10$ in univariate analysis and a stratification for cancer locations (digestive, breast and head and neck), DPD remained a significant strong and independent prognostic factor for OS, PFS and response $(p=$ 0.013, HR: 3.35 CI95\% [1.27-8.86]; $p<0.001$, HR: 3.15 CI95\% [1.75-5.66] and $p=0.033$, HR: 0.33 CI95\% [0.120.92] respectively). Supplementary data (Tables S1 to S3) provide detailed statistical results of multivariate regression analyses for OS, PFS and response and it shows that surgery associated to FP $(0.0017)$ and observed complete response during FP treatment $(<0.001)$ remained significantly related to OS. It was also put into evidence (Table S2) that other chemotherapy before FP $(<0.001)$, age at FP-based treatment (0.001) and number of FP cycles (0.006) were significantly associated to PFS. Disease severity (Table S3) at initial diagnostic (0.014) and other chemotherapy before FP (0.0049) were significantly linked to response.

Sensitivity analyses to evaluate the stability of multivariate models were tested firstly by removing 6 patient outliers from OS and PFS models and 5 patient outliers from response model. DPD activity remained significant with adjusted $p$-value at $0.001,<0.0001$ and 0.0064 for OS, PFS and response in multivariate models respectively. And in addition, by testing the same multivariate models only for patients with digestive cancer $(n=105)$, DPD activity was still significantly related to OS and PFS (adjusted p-value at 0.019 and 0.0007 respectively) and there was a strong tendency for a link with response $(p=0.075)$.

\section{Discussion}

From the present study it appears that high intrinsic DPD activity ( $\geq 0.30 \mathrm{nmol} / \mathrm{min} / \mathrm{mg}$ protein) is linked to 
Table 1 Patient and treatment characteristics versus DPD activity levels

\begin{tabular}{|c|c|c|c|c|}
\hline Variable & Modality & $\begin{array}{l}\text { Low DPD activity } \\
(<0.30 \mathrm{nmol} / \mathrm{min} / \mathrm{mg} \text { prot })\end{array}$ & $\begin{array}{l}\text { High DPD activity } \\
(\geq 0.30 \mathrm{nmol} / \mathrm{min} / \mathrm{mg} \text { prot })\end{array}$ & Fisher-test \\
\hline Age at FP-based treatment $(N A=0)$ & Mean [SD] & $64.22[12.11]$ & $64.38[13.67]$ & $0.95^{*}$ \\
\hline \multirow[t]{3}{*}{$\operatorname{Sex}(N A=0)$} & & & & 0.17 \\
\hline & Female & $67(85.9 \%)$ & $11(14.1 \%)$ & - \\
\hline & Male & $44(75.86 \%)$ & $14(24.14 \%)$ & - \\
\hline \multirow[t]{4}{*}{ Cancer location (NA = 0) } & & & & 0.76 \\
\hline & Digestive & $86(81.9 \%)$ & $19(18.1 \%)$ & - \\
\hline & Head and Neck & $5(71.43 \%)$ & $2(28.57 \%)$ & - \\
\hline & Breast & $20(83.33 \%)$ & $4(16.67 \%)$ & - \\
\hline \multirow[t]{3}{*}{ Disease severity $(\mathrm{NA}=0)$} & & & & 0.33 \\
\hline & Local disease & $35(87.5 \%)$ & $5(12.5 \%)$ & - \\
\hline & Advanced disease & $76(79.17 \%)$ & $20(20.83 \%)$ & - \\
\hline \multirow[t]{3}{*}{ DPD prescription (NA =0) } & & & & 0.59 \\
\hline & Before FP start & 89 (82.41\%) & 19 (17.59\%) & - \\
\hline & During FP Treatment & $22(78.57 \%)$ & $6(21.43 \%)$ & - \\
\hline \multirow[t]{3}{*}{ Other chemotherapy before FP (NA = } & & & & 0.61 \\
\hline & No & $85(82.52 \%)$ & $18(17.48 \%)$ & - \\
\hline & Yes & $26(78.79 \%)$ & $7(21.21 \%)$ & - \\
\hline \multirow[t]{4}{*}{ FP-based treatment $(N A=0)$} & & & & 0.61 \\
\hline & Capecitabine & $29(76.32 \%)$ & $9(23.68 \%)$ & - \\
\hline & $5-\mathrm{FU}$ & $58(82.86 \%)$ & $12(17.14 \%)$ & - \\
\hline & Both alternatively & $24(85.71 \%)$ & $4(14.29 \%)$ & - \\
\hline \multirow[t]{3}{*}{ FP-based toxicity $(\mathrm{NA}=4)$} & & & & 0.17 \\
\hline & No & $57(52.78 \%)$ & $16(69.57 \%)$ & - \\
\hline & Yes & $51(47.22 \%)$ & $7(30.43 \%)$ & - \\
\hline \multirow[t]{3}{*}{ Surgery associated to FP $(N A=1)$} & & & & 0.31 \\
\hline & No & $31(88.57 \%)$ & $4(11.43 \%)$ & - \\
\hline & Yes & 79 (79\%) & $21(21 \%)$ & - \\
\hline \multirow[t]{3}{*}{ Radiotherapy associated to FP (NA $=0$} & & & & 0.65 \\
\hline & No & $47(79.66 \%)$ & $12(20.34 \%)$ & - \\
\hline & Yes & $64(83.12 \%)$ & $13(16.88 \%)$ & - \\
\hline Nb. of chemotherapy lines $(N A=1)$ & Median [Min-Max] & $1[1-12]$ & $2[1-5]$ & $0.26^{* *}$ \\
\hline $\mathrm{Nb}$. of FP cycles $(\mathrm{NA}=0)$ & Median [Min-Max] & $7[1-48]$ & $9[1-18]$ & $0.39^{* *}$ \\
\hline \multicolumn{4}{|c|}{ Observed complete response during FP treatment $(N A=6)$} & 0.032 \\
\hline & No & $35(34.31 \%)$ & $14(60.87 \%)$ & - \\
\hline & Yes & $67(65.69 \%)$ & $9(39.13 \%)$ & - \\
\hline \multirow[t]{3}{*}{ Any recurrence $(\mathrm{NA}=0)$} & & & & 0.12 \\
\hline & No & $52(46.85 \%)$ & $7(28.0 \%)$ & - \\
\hline & Yes & $59(53.15 \%)$ & $18(72.0 \%)$ & - \\
\hline
\end{tabular}

NA not available data, FP Fluoropyrimidine, Fisher-test $p$-value Fisher exact test, ${ }^{*} p$-value Student-T test, ${ }^{* *} p$-value Wilcoxon test, SD Standard Deviation

OS, PFS and response in FP-treated patients. This is a well-established point that low DPD activity is a predictive risk factor for $\mathrm{FP}$-associated toxicity $[1,3,5-9]$. A potential benefit of DPYD genotype-guided FP-dose individualization has been recently reported [10]. French
Health Autoritaries (HAS) have provided strong recommendations to perform DPD deficiency screening on the basis of uracilemia determination before any treatment by FP with a guidance for dose reductions [17]. European authorities now consider that it is necessary to 


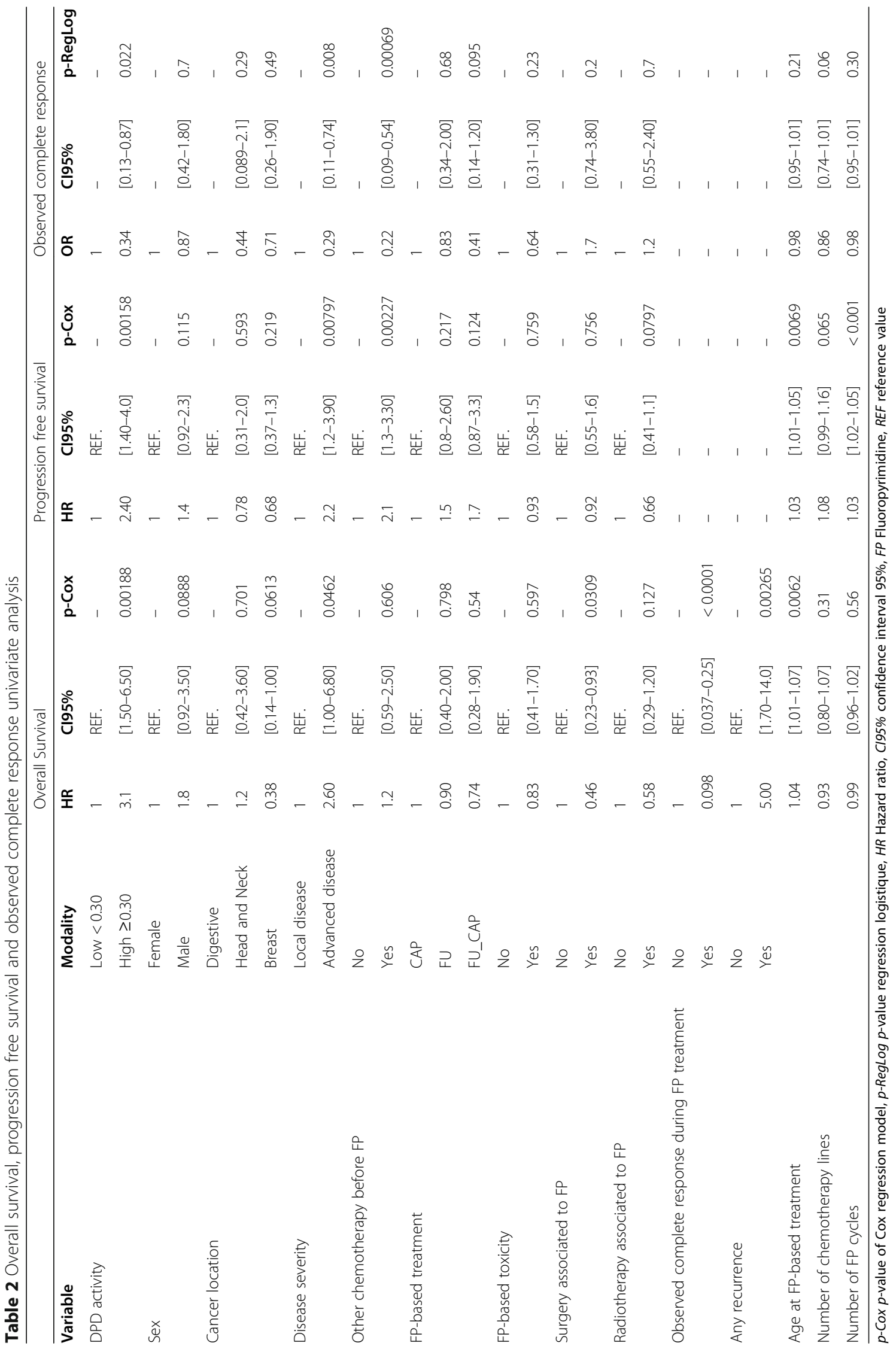



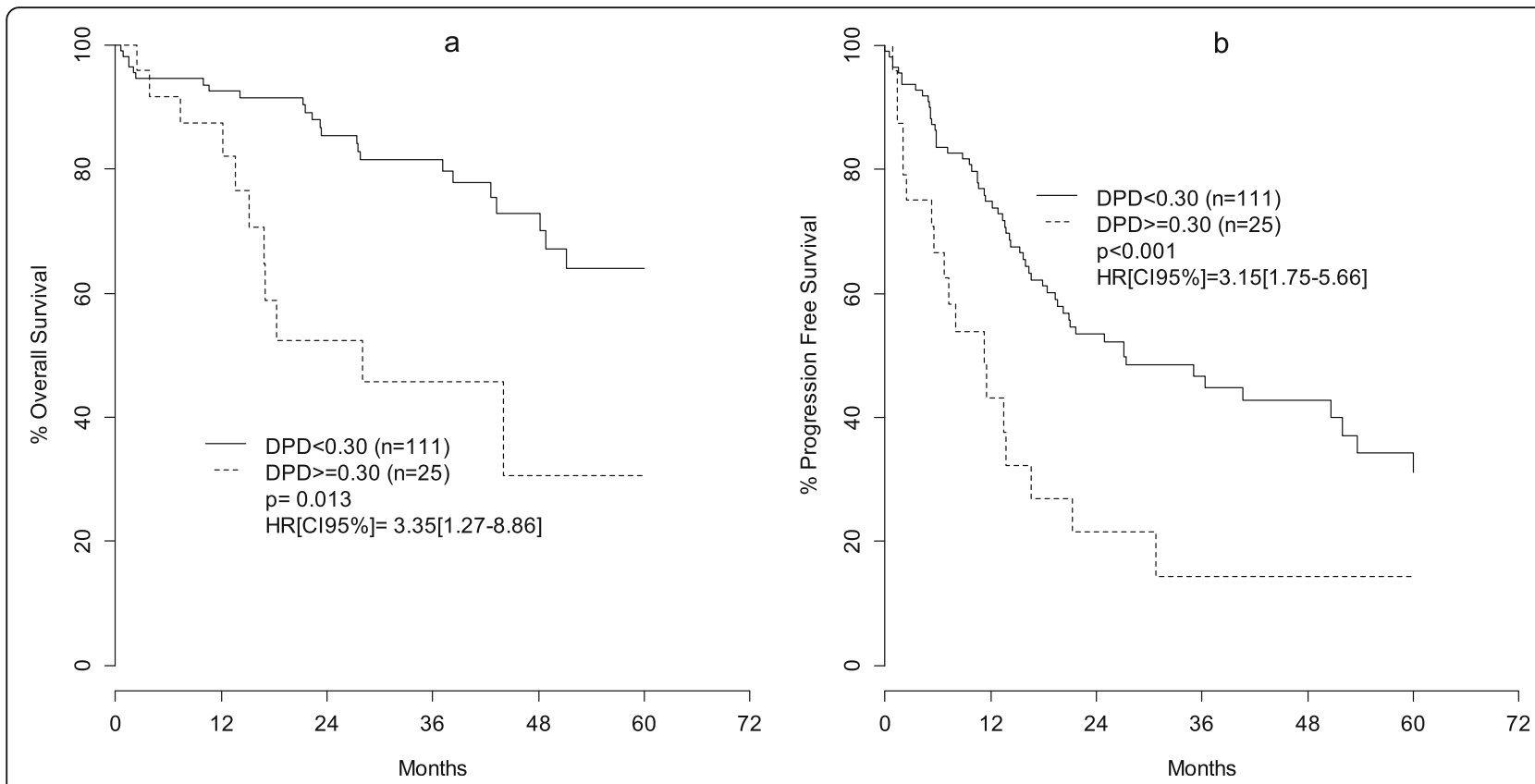

Fig. 1 a anb b Kaplan Meier survival curves for Overall and Progression Free Survival according to low $(<30)$ and high ( $\geq 30)$ DPD activity nmol/ $\mathrm{min} / \mathrm{mg}$ protein; $\mathrm{p}$ : adjusted $p$-value cox model; HR [Cl95\%]: adjusted hazard ratio [confidence interval 95\%]

perform an Eudra Vigilance analysis for reports of DPDFP-related toxicity. From our results, a new and complementary FP dosing strategy is pointing out. The fact that high individual DPD activity confers a particularly poor survival is new, as presently established in this retrospective series of 136 cancer patients.

Although different treatments regimens used in this study (capecitabine, 5-FU, both) could potentially mitigate the conclusions, all patients received a FP-based

Table 3 Univariate and multivariate analysis for overall survival, progression free survival and response according to DPD

\begin{tabular}{|c|c|c|}
\hline \multirow[t]{2}{*}{ Variable } & Univariate analysis & Multivariate analysis \\
\hline & $\mathrm{HR}$ [Cl95] & HR [Cl95] \\
\hline
\end{tabular}

\begin{tabular}{rllll}
\hline DPD activity versus overall survival $^{\mathrm{a}}$ & & \\
$<0.30$ & - & - & - & - \\
$\geq 0.30$ & 0.0018 & $3.1[1.50-6.50]$ & 0.013 & $3.35[1.27-8.86]$
\end{tabular}

DPD activity versus progression free survival ${ }^{a}$

$\begin{array}{lllll}<0.30 & - & - & - & - \\ \geq 0.30 & 0.0016 & 2.40[1.40-4.00] & <0.001 & 3.15[1.75-5.66]\end{array}$

DPD activity versus response to fluoropyrimidine ${ }^{\mathrm{b}}$

$<0.30$

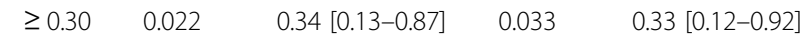

HR [C195] Hazard ratio and confidence interval 95\%

a Cox proportional hazards model

${ }^{\mathrm{b}}$ Logistic regression model

All variables associated with $p<0.10$ in univariate analysis were included in a Cox regression or Logistic regression model. All models were stratified on cancer locations (digestive, breast, head and neck) therapy. The distribution of DPD activity in the study population followed a Gaussian distribution comparable to those previously published and is thus representative of DPD activity profile in treated patients [2,3]. Behind the Gaussian shape of DPD activity lies in fact a myriad of impacting mutations $[6,18]$. In addition, there is the existence of a regulation of DPD gene expression at a transcriptional level $[19,20]$. Of note, as recently been reported, there is the presence of rare mutations which confer high intrinsic DPD activity [1]. Theoretically, germinal DPD status can impact DPD expression at tumoral level. However, the fact that $\mathrm{Tp} 53$ has recently been shown to play an important role in controlling pyrimidine catabolism through repression of DPD expression at tumoral level may moderate this view [21]. A marked variability in intra-tumoral DPD expression was previously reported to be linked to the occurrence of resistance to FP therapy, at both experimental [11] and clinical levels [14]. Our previous study [22] indicated that both thymidylate synthase and DPD are overexpressed in deficient mismatch repair dMMR tumours, thus providing possible explanation for a relative resistance of MMR tumours to FP-based therapy [23]. More recently, in a trial randomized to either gemcitabine or 5-FU plus folinic acid in pancreatic carcinoma, Elander et al. [24] showed that high intra-tumoral DPD expression was a negative prognostic biomarker and that low DPD tumor expression indicated better prognosis, at least for patients treated with 5-FU. 
Therefore, at least in part, high intrinsic DPD activity (as here measured in PBMC) may reflect DPD activity in tumors and influence disease outcome following FP therapy. It is also clear that the impact of high intrinsic DPD activity is pharmacokinetic by nature with, consequently, relatively low levels of circulating FP resulting from high global DPD activity, a phenomenon occurring in the liver. In line with this view is the previously reported link between patient survival and 5-FU circulating exposure $[25,26]$. The existence of a significant correlation between DPD activity in normal liver tissue and lymphocytes [27] supports the extrapolation between lymphocyte DPD activity and FP pharmacokinetic behavior with clinical consequences. Thus, although the present study did not incorporate FU pharmacokinetic analyses, one can estimate that abnormally high DPD activity may be translated into low FP circulating levels, leading to a relative loss of treatment efficacy, at least for iv-FP keeping in mind that capecitabine delivers FU at the target cellular level itself.

Ideally, an intrinsic prognostic role for DPD activity should be tested in patients not receiving FP. This appears justified because it has recently been shown that a nucleotide imbalance exists across different cancer types. This imbalance can potentially be introduced by abnormal DPD activity and a pyrimidine/purine disequilibrium may increase mutagenesis $[28,29]$ and consequently may influence disease outcome [29].

The present exploratory study has several limitations. This is a retrospective and heterogeneous cohort study including digestive, breast and head \& neck FP-treated cancer patients receiving multifactorial treatment like intravenous 5-FU, oral capecitabine or both. To avoid most of the biases in the analyses, all multivariate analysis have been stratified on cancer location and these analyses were also adjusted on all variables that could be confounding factors. Moreover, the same statistically significant results were found when analyses were made on digestive cancer patients only.

\section{Conclusions}

Above all, the present report may confer practical implications in the current era of precision medicine in cancer. Hence, at the light of the present data, DPD activity screening could result in a two-pronged approach: FP dose reduction in case of low intrinsic DPD activity and, inversely, an increased FP dose for high intrinsic DPD activity (Fig. 2). This view contrasts with the caricatured current strategy consisting in reducing FP dosage in cases of DPD abnormality (on a phenotypic and/or a genotypic basis) potentially diminishing the ability to clear 5-FU from the body [18]. To ignore cases with

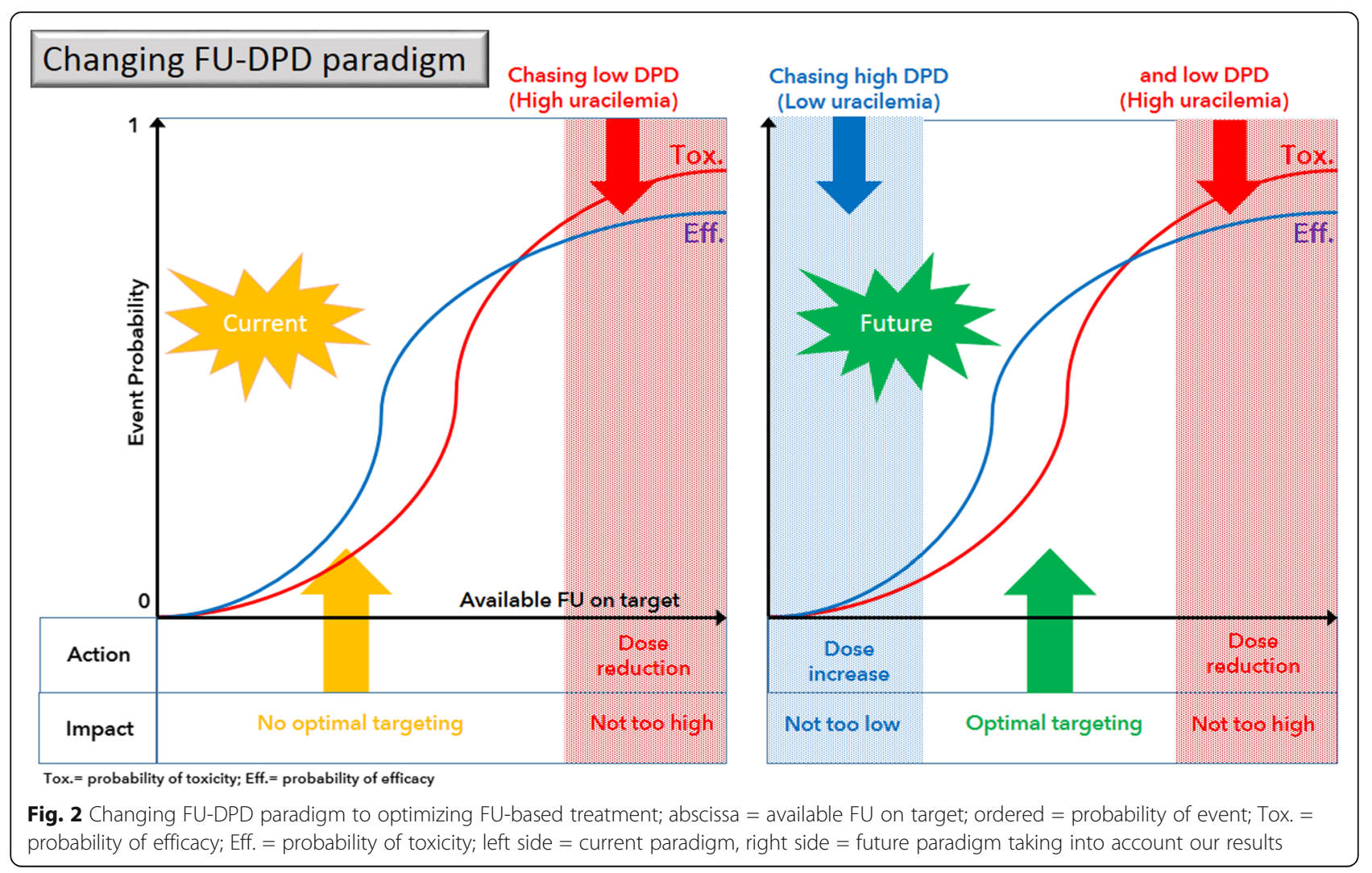


relatively high intrinsic DPD activity could confer a risk for a loss of treatment success. In final, the present study contributes to broaden the strategy regarding DPD screening. Such a new strategy needs to be prospectively validated for setting a true personalized DPD-based treatment including pharmacokinetics analyses. In line with this view, our group is currently conducting a multicenter clinical study (DPD-MAX trial $\mathrm{N}^{\circ}$ Eudract 2017-002037-31) aiming at establishing a more reliable prospective validation of the link between high intrinsic DPD activity and loss of FP treatment efficacy.

\section{Supplementary information}

Supplementary information accompanies this paper at https://doi.org/10. 1186/s12885-020-06907-0.

Additional file 1: Table S1. Overall survival multivariate analysis. Table S2. Progression free survival multivariate analysis. Table S3. Observed complete response during FP treatment multivariate analysis.

Additional file 2: Fig. S1. Smoothing spline and C195\% fit to DPD activity $\mathrm{nmol} / \mathrm{min} / \mathrm{mg}$ protein versus overall survival and $95 \%$ confident interval. Fig. S2. Smoothing spline fit and C195\% to DPD activity nmol/ $\mathrm{min} / \mathrm{mg}$ protein versus progression free survival and 95\% confident interval. Fig. S3. Smoothing spline and IC95\% fit to DPD activity nmol/ $\mathrm{min} / \mathrm{mg}$ protein versus observed complete response during FP treatment.

\section{Abbreviations}

DPD: DihydroPyrimidine Dehydrogenase; FP : FluoroPyrimidine; 5-FU: 5 Fluorouracyl; C195\% : 95\% Confident Interval; HPLC: High-Performance Liquid Chromatography; CR: Complete Response; HAS: French Health Autoritaries; OS: Overall Survival; PFS: Progression Free Survival; PBMC: Peripheral Blood Mononuclear Cell; HR: Hazard Ratio

\section{Acknowledgements}

Not applicable

\section{Authors' contributions}

All authors have read and approved the manuscript. EC: statistical design, analyses and major contributor in writing the manuscript. EF: inclusion of patients. MCE: pharmacology consultant. JMF, FP, EB, AB, RL, OC: inclusion of patients. JV: statistical consultant. RS: data management. GM: pharmacology analyses and major contributor in writing the manuscript.

\section{Funding}

No funding.

\section{Availability of data and materials}

The datasets during and/or analysed during the current study available from the corresponding author on reasonable request.

\section{Ethics approval and consent to participate}

This study was declared to the Commission Nationale Informatique et Liberté (CNIL N¹7002) and all patients agreed that their data could be used for retrospective biomedical study.

\section{Consent for publication}

Not applicable.

\section{Competing interests}

The authors declare that they have no competing interests.

\section{Author details}

${ }^{1}$ Centre Antoine-Lacassagne, Epidemiology and Biostatistics Unit, Université Côte d'Azur, 33 Avenue de Valombrose, 06189 Nice Cedex 2, Nice, France. ${ }^{2}$ Centre Antoine-Lacassagne, Medical Oncology, Université Côte d'Azur, Nice, France. ${ }^{3}$ Centre Antoine-Lacassagne, Oncopharmacology Unit, Université
Côte d'Azur, Nice, France. ${ }^{4}$ Centre Antoine-Lacassagne, Surgical Oncology, Université Côte d'Azur, Nice, France. ${ }^{5}$ Centre Antoine Lacassagne, Institut Universitaire de la Face et du Cou, Head and Neck Surgery, Université Côte d'Azur, Nice, France.

Received: 2 December 2019 Accepted: 27 April 2020

Published online: 18 May 2020

\section{References}

1. Offer SM, Fossum CC, Wegner NJ, Stuflesser AJ, Butterfield GL, Diasio RB. Comparative functional analysis of DPYD variants of potential clinical relevance to dihydropyrimidine dehydrogenase activity. Cancer Res. 2014; 74(9):2545-54

2. Etienne MC, Lagrange $J$, Dassonville $O$, Fleming R, Thyss A, Renee N, et al. Population study of dihydropyrimidine dehydrogenase in cancer patients. J Clin Oncol. 1994;12(11):2248-53.

3. Lu Z, Zhang R, Diasio RB. Dihydropyrimidine dehydrogenase activity in human peripheral blood mononuclear cells and liver: population characteristics, newly identified deficient patients, and clinical implication in 5-fluorouracil chemotherapy. Cancer Res. 1993:53(22):5433-8.

4. van Kuilenburg ABP, Meijer J, Meinsma R, Perez-Duenas B, Alders M, Bhuiyan ZA, et al. Dihydropyrimidine dehydrogenase deficiency: Homozygosity for an extremely rare variant in DPYD due to Uniparental Isodisomy of chromosome 1. JIMD Rep. 2019:45:65-9.

5. Etienne-Grimaldi MC, Boyer JC, Beroud C, Mbatchi L, van Kuilenburg A, Bobin-Dubigeon C, et al. New advances in DPYD genotype and risk of severe toxicity under capecitabine. PLoS One. 2017;12(5):e0175998.

6. Meulendijks D, Henricks LM, Sonke GS, Deenen MJ, Froehlich TK, Amstutz U, et al. Clinical relevance of DPYD variants C.1679T>G, c.1236G>a/HapB3, and c.1601G >a as predictors of severe fluoropyrimidine-associated toxicity: a systematic review and meta-analysis of individual patient data. Lancet Oncol. 2015;16(16):1639-50.

7. van Kuilenburg AB, Meijer J, Maurer D, Dobritzsch D, Meinsma R, Los M, et al. Severe fluoropyrimidine toxicity due to novel and rare DPYD missense mutations, deletion and genomic amplification affecting DPD activity and mRNA splicing. Biochim Biophys Acta. 2017;1863(3):721-30.

8. Wettergren Y, Carlsson G, Odin E, Gustavsson B. Pretherapeutic uracil and dihydrouracil levels of colorectal cancer patients are associated with sex and toxic side effects during adjuvant 5-fluorouracil-based chemotherapy. Cancer. 2012;118(11):2935-43.

9. van Kuilenburg AB. Dihydropyrimidine dehydrogenase and the efficacy and toxicity of 5-fluorouracil. Eur J Cancer. 2004;40(7):939-50.

10. Henricks LM, Lunenburg C, de Man FM, Meulendijks D, Frederix GWJ, Kienhuis $\mathrm{E}$, et al. DPYD genotype-guided dose individualisation of fluoropyrimidine therapy in patients with cancer: a prospective safety analysis. Lancet Oncol. 2018;19(11):1459-67.

11. Beck A, Etienne MC, Cheradame S, Fischel JL, Formento P, Renee N, et al. A role for dihydropyrimidine dehydrogenase and thymidylate synthase in tumour sensitivity to fluorouracil. Eur J Cancer. 1994;30A(10):1517-22.

12. Etienne MC, Cheradame S, Fischel JL, Formento P, Dassonville O, Renee N, et al. Response to fluorouracil therapy in cancer patients: the role of tumoral dihydropyrimidine dehydrogenase activity. J Clin Oncol. 1995;13(7): 1663-70.

13. Milano G, McLeod HL. Can dihydropyrimidine dehydrogenase impact 5fluorouracil-based treatment? Eur J Cancer. 2000;36(1):37-42.

14. Zhang C, Liu H, Ma B, Song Y, Gao P, Xu Y, et al. The impact of the expression level of Intratumoral Dihydropyrimidine dehydrogenase on chemotherapy sensitivity and survival of patients in gastric Cancer: a metaanalysis. Dis Markers. 2017;2017:9202676.

15. Harris BE, Song R, Soong SJ, Diasio RB. Relationship between dihydropyrimidine dehydrogenase activity and plasma 5-fluorouracil levels with evidence for circadian variation of enzyme activity and plasma drug levels in cancer patients receiving 5 -fluorouracil by protracted continuous infusion. Cancer Res. 1990;50(1):197-201.

16. Harris BE, Song RL, He YJ, Soong SJ, Diasio RB. Circadian rhythm of rat liver dihydropyrimidine dehydrogenase. Possible relevance to fluoropyrimidine chemotherapy. Biochem Pharmacol. 1988:37(24):4759-62

17. INCA HADSHIdC. Recherche de déficit en dihydropyrimidine déshydrogénase en vue de prévenir certaines toxicités sévères survenant sous traitement comportant des fluoropyrimidines. HAS. 2018. 
18. Amstutz U, Henricks LM, Offer SM, Barbarino J, Schellens JHM, Swen JJ, et al. Clinical Pharmacogenetics implementation consortium (CPIC) guideline for Dihydropyrimidine dehydrogenase genotype and Fluoropyrimidine dosing: 2017 update. Clin Pharmacol Ther. 2018:103(2):210-6.

19. Offer SM, Butterfield GL, Jerde CR, Fossum CC, Wegner NJ, Diasio RB. microRNAs miR-27a and miR-27b directly regulate liver dihydropyrimidine dehydrogenase expression through two conserved binding sites. Mol Cancer Ther. 2014;13(3):742-51.

20. Wu R, Nie Q, Tapper EE, Jerde CR, Dunlap GS, Shrestha S, et al. Histone H3K27 Trimethylation modulates 5-fluorouracil resistance by inhibiting PU.1 binding to the DPYD promoter. Cancer Res. 2016;76(21):6362-73.

21. Gokare P, Finnberg NK, Abbosh PH, Dai J, Murphy ME, El-Deiry WS. P53 represses pyrimidine catabolic gene dihydropyrimidine dehydrogenase (DPYD) expression in response to thymidylate synthase (TS) targeting. Sci Rep. 2017;7(1):9711.

22. Etienne-Grimaldi MC, Mahamat A, Chazal M, Laurent-Puig P, Olschwang S, Gaub MP, et al. Molecular patterns in deficient mismatch repair colorectal tumours: results from a French prospective multicentric biological and genetic study. Br J Cancer. 2014;1 10(11):2728-37.

23. Sinicrope FA, Foster NR, Thibodeau SN, Marsoni S, Monges G, Labianca R, et al. DNA mismatch repair status and colon cancer recurrence and survival in clinical trials of 5-fluorouracil-based adjuvant therapy. J Natl Cancer Inst. 2011;103(11):863-75.

24. Elander NO, Aughton K, Ghaneh P, Neoptolemos JP, Palmer DH, Cox TF, et al. Expression of dihydropyrimidine dehydrogenase (DPD) and hENT1 predicts survival in pancreatic cancer. Br J Cancer. 2018;118(7):947-54.

25. Gamelin E, Delva R, Jacob J, Merrouche Y, Raoul JL, Pezet D, et al. Individual fluorouracil dose adjustment based on pharmacokinetic follow-up compared with conventional dosage: results of a multicenter randomized trial of patients with metastatic colorectal cancer. J Clin Oncol. 2008;26(13): 2099-105.

26. Milano G, Etienne MC, Renee N, Thyss A, Schneider M, Ramaioli A, et al. Relationship between fluorouracil systemic exposure and tumor response and patient survival. J Clin Oncol. 1994;12(6):1291-5.

27. Chazal M, Etienne MC, Renee N, Bourgeon A, Richelme H, Milano G. Link between dihydropyrimidine dehydrogenase activity in peripheral blood mononuclear cells and liver. Clin Cancer Res. 1996;2(3):507-10.

28. Schmidt TT, Reyes G, Gries K, Ceylan CU, Sharma S, Meurer M, et al. Alterations in cellular metabolism triggered by URA7 or GLN3 inactivation cause imbalanced dNTP pools and increased mutagenesis. Proc Natl Acad Sci U S A. 2017;114(22):E4442-E51.

29. Lee JS, Adler L, Karathia H, Carmel N, Rabinovich S, Auslander N, et al. Urea cycle Dysregulation generates clinically relevant genomic and biochemical signatures. Cell. 2018;174(6):1559-70 e22.

\section{Publisher's Note}

Springer Nature remains neutral with regard to jurisdictional claims in published maps and institutional affiliations.

Ready to submit your research? Choose BMC and benefit from:

- fast, convenient online submission

- thorough peer review by experienced researchers in your field

- rapid publication on acceptance

- support for research data, including large and complex data types

- gold Open Access which fosters wider collaboration and increased citations

- maximum visibility for your research: over $100 \mathrm{M}$ website views per year

At $\mathrm{BMC}$, research is always in progress.

Learn more biomedcentral.com/submissions 